vs $44.6 \%$ in the 2 nd group. The hospital stay was significantly less: in the 1st group: $18.8 \pm 1.4$ days vs $26.9 \pm 4.6$ days in the 2 nd group. The recurrence rate in the 1 st group was $9.53 \%$ vs $24.6 \%$ in the 2 nd group, which is probably due to wider excision of the perineal tissue using reconstructive plastic surgery. Five-year survival was $76.5 \%$ in the 1 st vs $56 \%$ in the 2nd group.

Conclusion The use of reconstructive plastic surgery for closing of wound defects after radical vulvectomy reduces the incidence of postoperative complications and improves oncological treatment results.

Disclosures None.

\section{IMPACT OF TUMOUR-FREE MARGIN AND LYMPH NODE RATIO ON ONCOLOGIC OUTCOMES IN VULVAR CANCER- A SINGLE INSTITUTE EXPERIENCE}

Chinmoyee Kalita, Shruti Bhatia, Renuka Gupta. Action Cancer Hospital; Gynaecological Oncology

\subsection{6/ijgc-2020-ESG0.188}

Introduction/Background Vulvar cancers accounts for 3-5\% of all gynaecological malignancies. Inguinal lymph node involvement and tumour-free margin are considered as significant prognostic factors for survival in patients with vulvar cancer. Surgery is the cornerstone of treatment. Lymph node ratio (LNR) is the ratio of the number of positive lymph nodes (LN) to the number of removed LN. This parameter incorporates not only the burden of nodal disease and cancer spread but also the extent and quality of surgical staging. Current data in the literature regarding a minimum oncologically safe tumour-free margin distance are contradictory. The objective of this study was to evaluate the association of tumour-free margin and LNR with oncologic outcomes in vulvar cancer.

Methodology Retrospective analysis evaluating 21 patients of vulvar squamous cell cancer who underwent primary surgery at our institution from January 2013 to December 2018. Patients were stratified into three risk groups according to tumour-free margin $(<5 \mathrm{~mm}, \geq 5 \mathrm{~mm}-<8 \mathrm{~mm}$ and $\geq 8$ $\mathrm{mm})$ and LNR $(0 \%,>0-20 \%$ and $>20 \%)$ to compare oncologic outcomes. Follow up was done till August 2019. Qualitative variables were correlated using Chi-Square test/Fisher's exact test. Overall survival (OS), disease free survival (DFS) and recurrence rate (RR) were estimated by Kaplan-Meier method. Log rank test was used for comparison among the groups.

Results Median age was 67 years. Median DFS and OS were 17.4 months and 27.7 months respectively. 11 patients (52.4\%) developed recurrence of which 8 had local recurrence. RR in tumour-free margin $<5 \mathrm{~mm}$ group was high $(100 \%)$ as compared to $\geq 5 \mathrm{~mm}-<8 \mathrm{~mm}(50 \%)$ and $\geq 8$ $\mathrm{mm}(30 \%)$ groups $(\mathrm{p}=0.037)$. DFS rates at the end of the study were increasing from $0.0 \%$ (in $<5 \mathrm{~mm}$ group) to $66.7 \%$ (in $\geq 8 \mathrm{~mm}$ group) and as well OS rates also (50\% to $65.6 \%)$. At the end of the study DFS rates in patients with LNR $0 \%,>0-<20 \%$ and $\geq 20 \%$ were $57.1 \%, 22.5 \%$ and $0.0 \%$ respectively $(\mathrm{p}=0.047)$. On applying Log rank test no significant difference was seen in the OS between the different groups of LNR.
Conclusion Prognosis of vulvar cancer patient is affected by tumour-free margin and high LNR in our study. DFS is significantly reduced in patients with tumour-free margin $<5$ $\mathrm{mm}$ even in the absence of LN metastasis. High LNR is associated with unfavourable DFS. Tumour-free margin $\geq 8$ $\mathrm{mm}$ is a good prognostic factor in patients of vulvar carcinoma.

Disclosures This study received no external funding and sponsorship. The authors declare no conflict of interest.

\section{RISK FACTORS AFFECTING ONCOLOGICAL OUTCOMES IN VULVAR CANCER UNDERGOING PRIMARY SURGERY: CASE SERIES FROM A TERTIARY CANCER CENTRE}

${ }^{1}$ Ts Shylasree, ${ }^{2}$ Neha Kumar, 'Ushashree Das, ${ }^{1}$ Amita Maheswari, ${ }^{3}$ Lavanya Gurram, ${ }^{4}$ Supriya Chopra, ${ }^{3}$ Gargee Mulye, ${ }^{5}$ Santosh Menon, ${ }^{5}$ Bharat Rekhi, ${ }^{5}$ Kedar Deodhar, ${ }^{6}$ Umesh Mahantshetty. 'Tata Memorial Hospital; Department of Gynecologic Oncology and Mdt; ${ }^{2}$ Blk Superspeciality Hospital; Gynaec Oncology; ${ }^{3}$ Tata Memorial Hospital; Radiation Oncology; ${ }^{4}$ Actrec, Tata Memorial Centre; ${ }^{5}$ Tata Memorial Hospital, Pathology, Mumbai, India; ${ }^{6}$ Homi Bhabha Cancer Hospital, Tata Memorial Centre; Radiation Oncology

\subsection{6/ijgc-2020-ESG0.189}

Introduction/Background To evaluate risk factors associated with adverse oncological outcomes in women undergoing primary surgery for vulvar cancer.

Methodology Eighty-one patients who underwent primary surgery for SCC vulva and were registered at tertiary cancer hospital between January 2011- December 2018 were analysed retrospectively. Adverse risk factors such as age, stage, tumour free margins (TFM), depth of stromal invasion (DSI) and lymph node status were analysed using univariate analysis and survival was calculated using Kaplan-Meir curves.

Results Median age was 55 years. All patients underwent either wide radical excision/radical vulvectomy. Groins were addressed in 63 patients. Median follow up was 42 months. Overall survival and DFS at 3 years were $82 \%$ and $69 \%$ respectively. On univariate analysis of 81 patients, DSI, TFM $(<10 \mathrm{~mm})$ and stage III $>$ had statistically significant effect on DFS, whereas DSI and stage III $>$ had statistically significant effect on overall survival. Age $>60$ years did not have significant effect on oncological outcomes.

Following surgery, based on final histology report, 63 patients remained within stage I/II, whereas 18 patients were upstaged to Stage III \& above (Stage III $>$ ). Stage migration was mainly due to lymph node positivity on histology.

On subgroup analysis of 63 patients in good prognostic group (stage 1/II), DFS and TFM had statistically significant adverse effects on DFS and OS. Overall survival at 3 years was $86 \%$ and DFS $78 \%$ in this subgroup.

Of 18 patients in poor prognostic subgroup (post-surgical stage III and above), 1 in 3 developed recurrence and 1 in 2 died of disease.

Conclusion There is a positive correlation of DSI and lymphnode metastasis, hence lymphadenectomy is proposed based on DSI subcategory in FIGO stage I. Our study found DSI as an independent risk factor which affects both DFS and overall survival in early stage vulvar cancer with negative lymph nodes.

Disclosures None. 